\title{
Status Update on DLR's OSIRIS program
}

Christian Fuchs et. al

Institute of Communications and Navigation German Aerospace Center (DLR) 


\section{OSIRIS: Optical Space Infrared Downlink System}

- Development of experimental optical terminals optimized for small satellites (from Cubesats to $\sim 100 \mathrm{~kg}$ )

- Wavelength: $1550 \mathrm{~nm}$ range

- Supplement for GEO-relay systems, as e.g. EDRS

- Enabler for exhaustive channel measurements @ 1550 nm

- Usage and qualification of COTS-Components

- Current Status

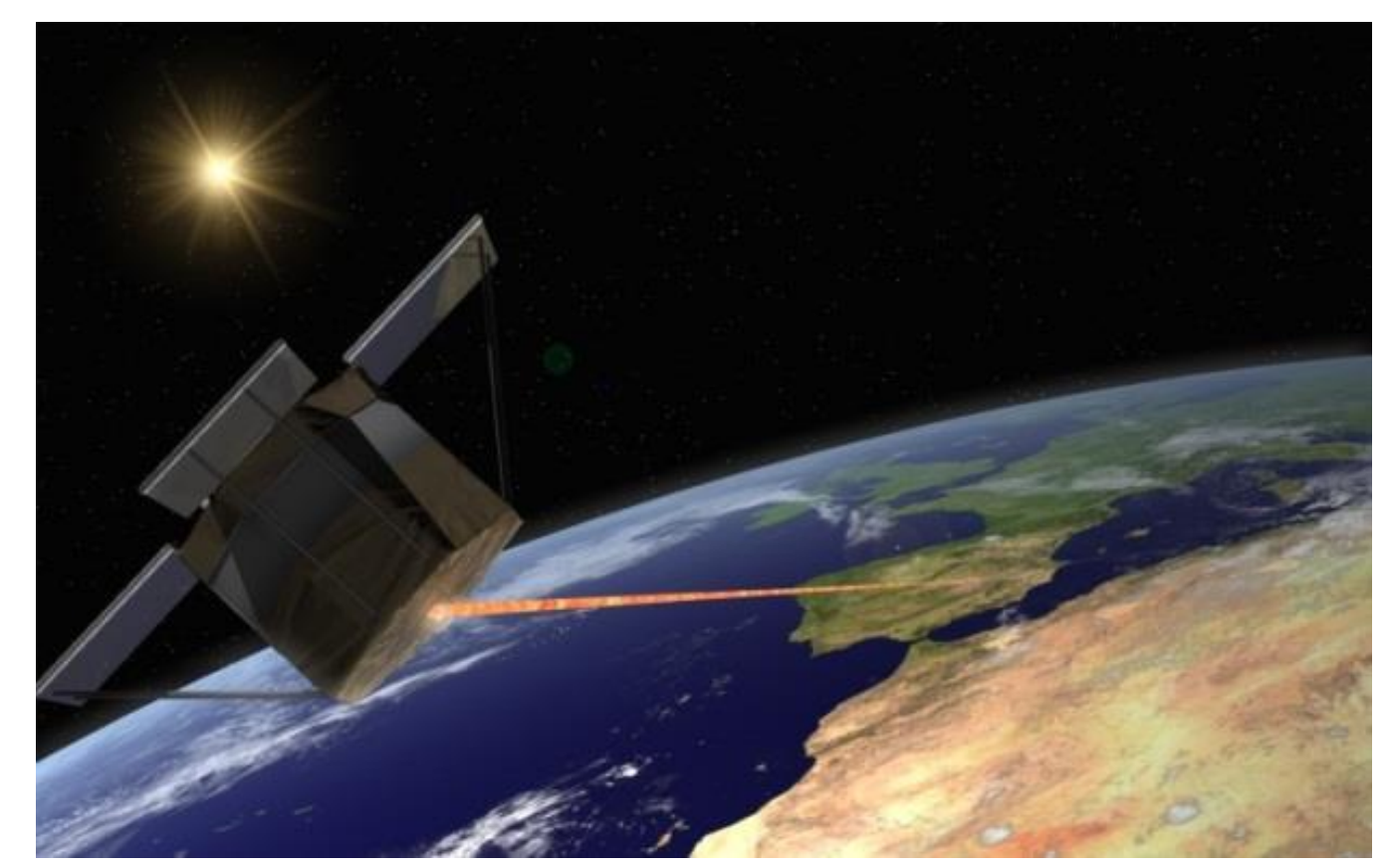

- Two generations of flight models finished \& in Space

- Further terminals currently under development

- Six OSIRIS-terminals in Space by 2020 


\section{OSIRIS development roadmap}

\section{OSIRISv2:}

Closed-Loop Body Pointing

Data rate: up to $1 \mathrm{Gbit} / \mathrm{s}$

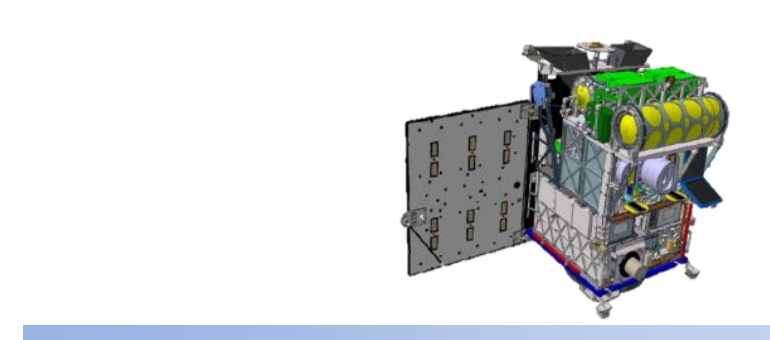

2018

2017

2016

\section{OSIRIS4CubeSat:}

Active Beam Steering combined

with body pointing

Data rate: up to $100 \mathrm{Mbit} / \mathrm{s}$

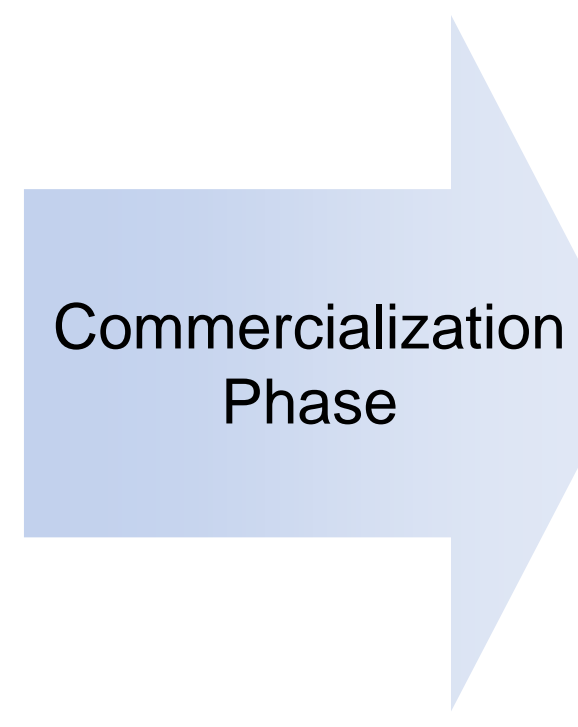

OSIRISv3:

OSIRISv1:

Open-Loop Body Pointing Data rate: up to $200 \mathrm{Mbit} / \mathrm{s}$

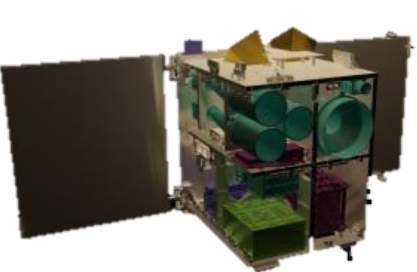

2019
Active Beam Steering with Coarse Pointing Assembly Data rate: up to $10 \mathrm{Gbit} / \mathrm{s}$
Quantum Comm.

Experiments

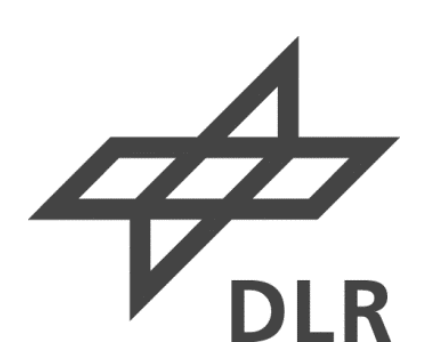

O TESAT
Quantum Key

Distribution 


\section{OSIRIS-generations one and two}

\section{1st OSIRIS generation}
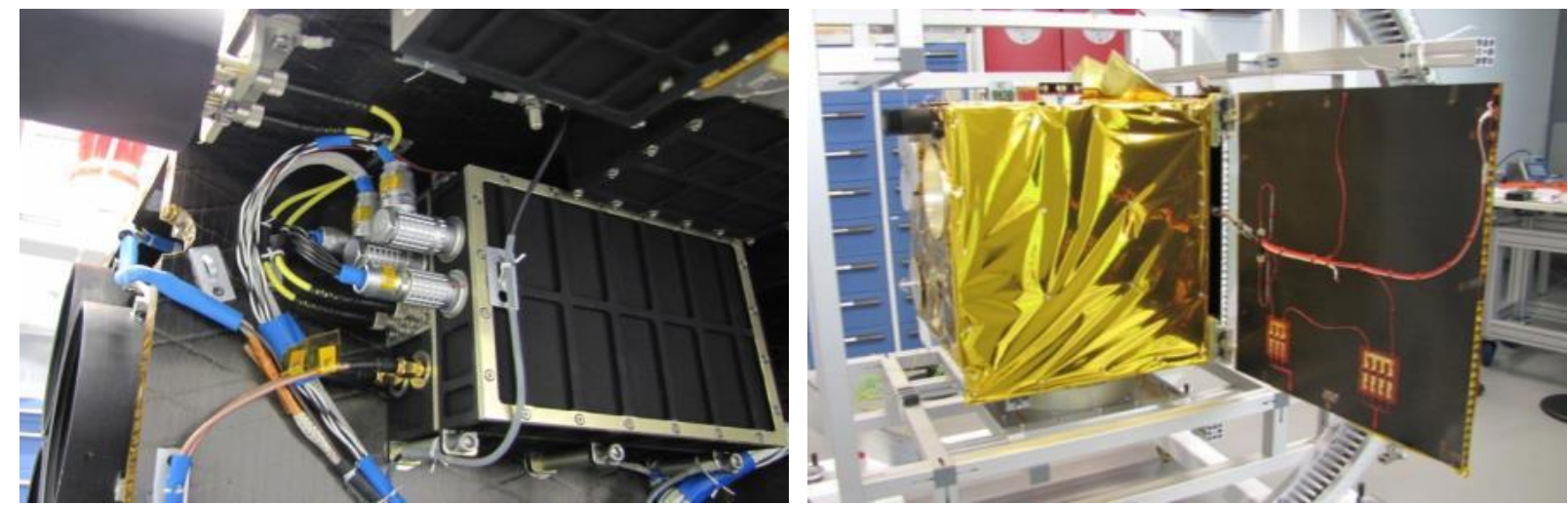

- $1.34 \mathrm{~kg}, 25 \mathrm{~W}, \sim 200 \mathrm{Mbps}$

- Flying Laptop satellite (Univ. of Stuttgart)

- Open-loop pointing of satellite

- Launch: 14th of July, 2017

\section{2nd OSIRIS generation}
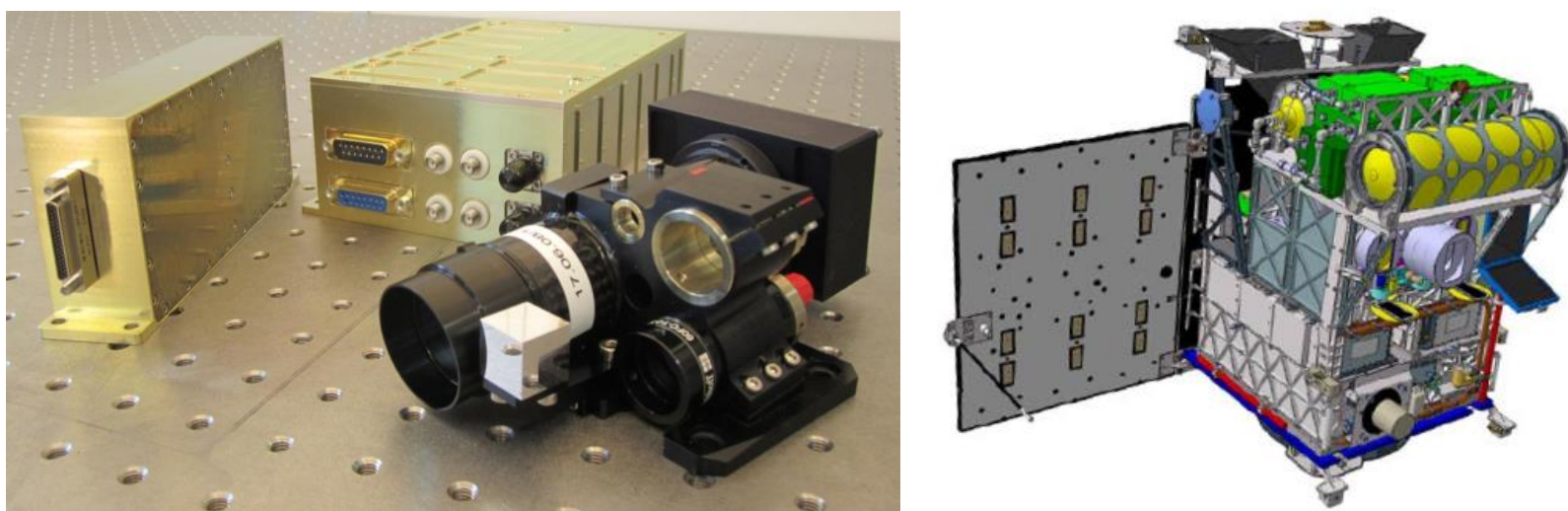

- $1.65 \mathrm{~kg}, 37 \mathrm{~W}, \sim 1$ Gbps

- BIROS satellite (DLR OS / Berlin)

- Closed-loop pointing of satellite

- Launch: 20th of June, 2016 


\section{Current OSIRISv1 + v2 status}

- Both Flying Laptop and BIROS are in good health

- First tests have been performed with Optical Ground Stations of DLR and ESA

- Telemetries of OSIRIS laser sources and tracking sensor show good results

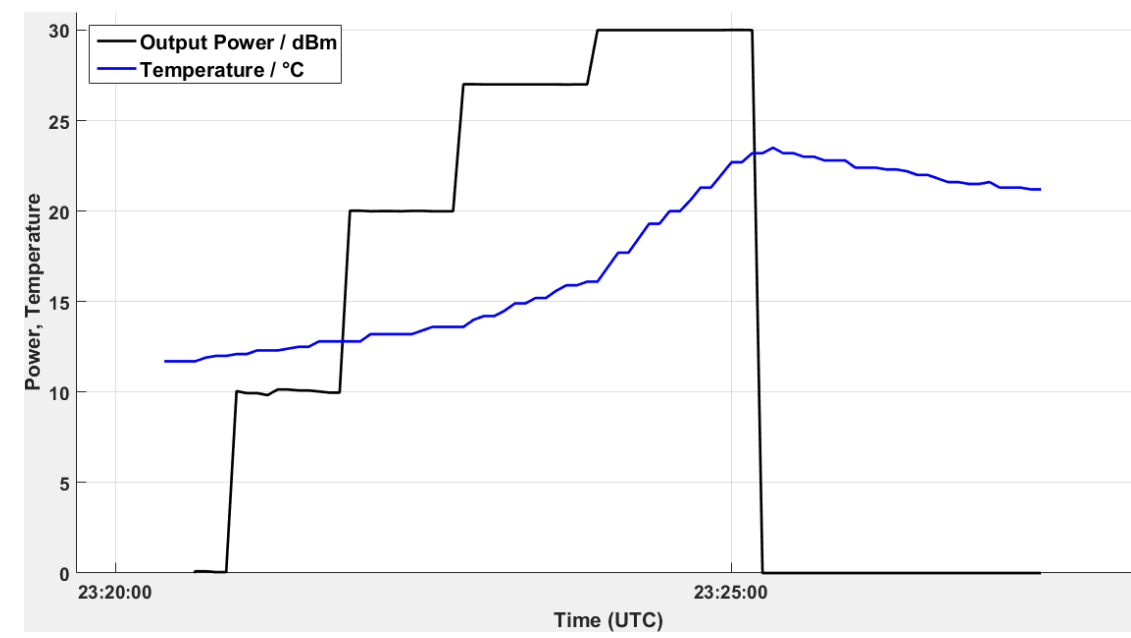

Telemetry of OSIRISv1-EDFA (operated in Space)

$\rightarrow$ OSIRISv1 and OSIRISv2 telemetry shows that both systems work as expected

- Stable reception of OSIRISv1 signals $\rightarrow$ next slides

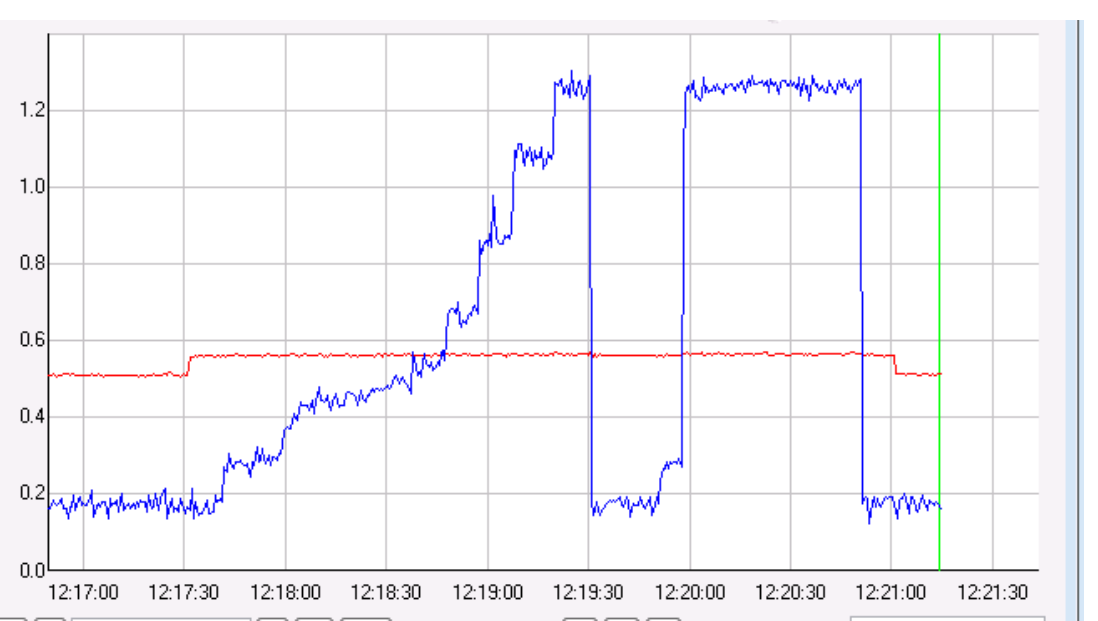

Telemetry of OSIRISv2-EDFA (operated in Space) 


\section{First results from Flying Laptop}

- First „flash“ from Flying Laptop during search pattern
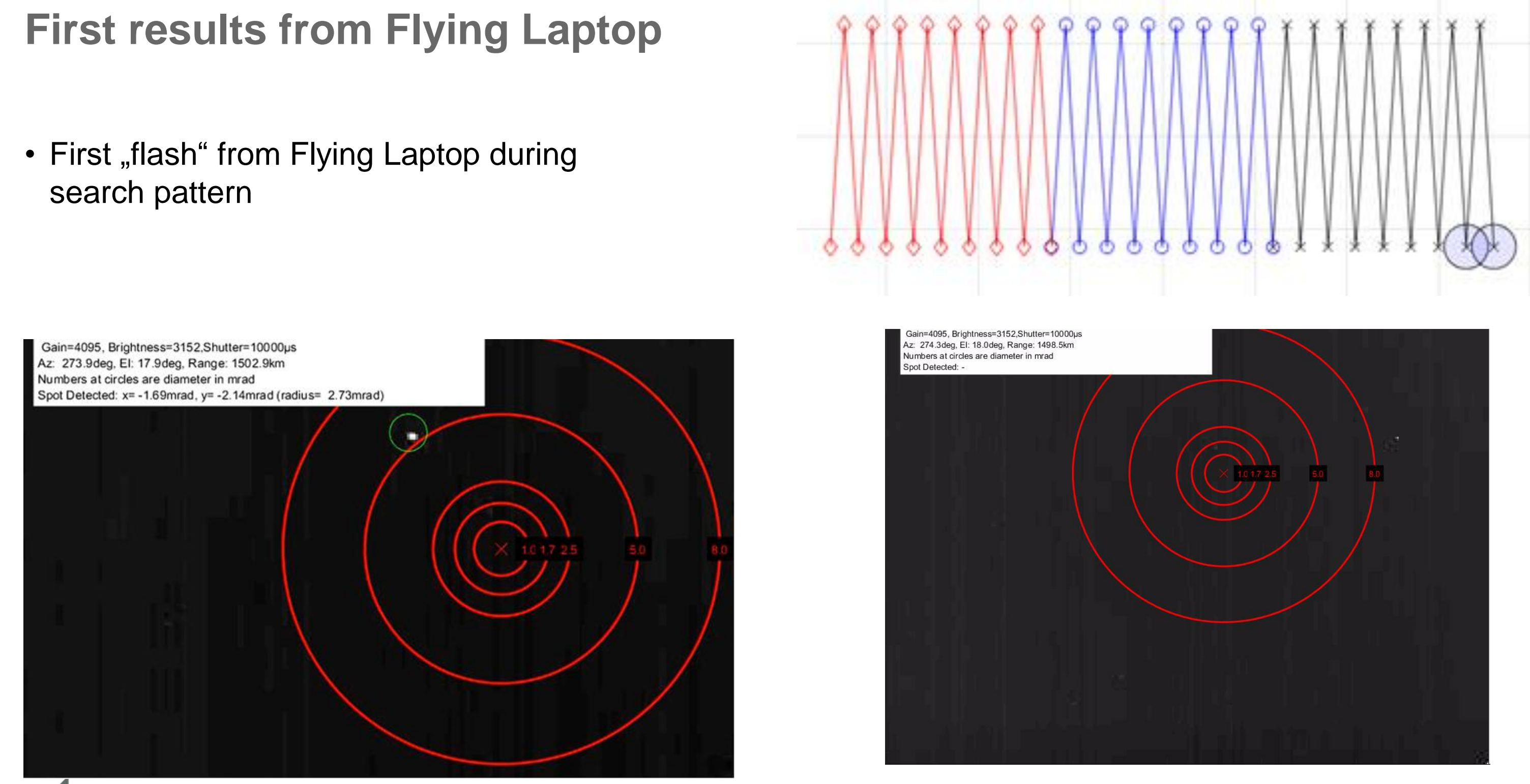
Flying Laptop - Signal reception after offset optimization Acquisition at 0.4 deg elevation

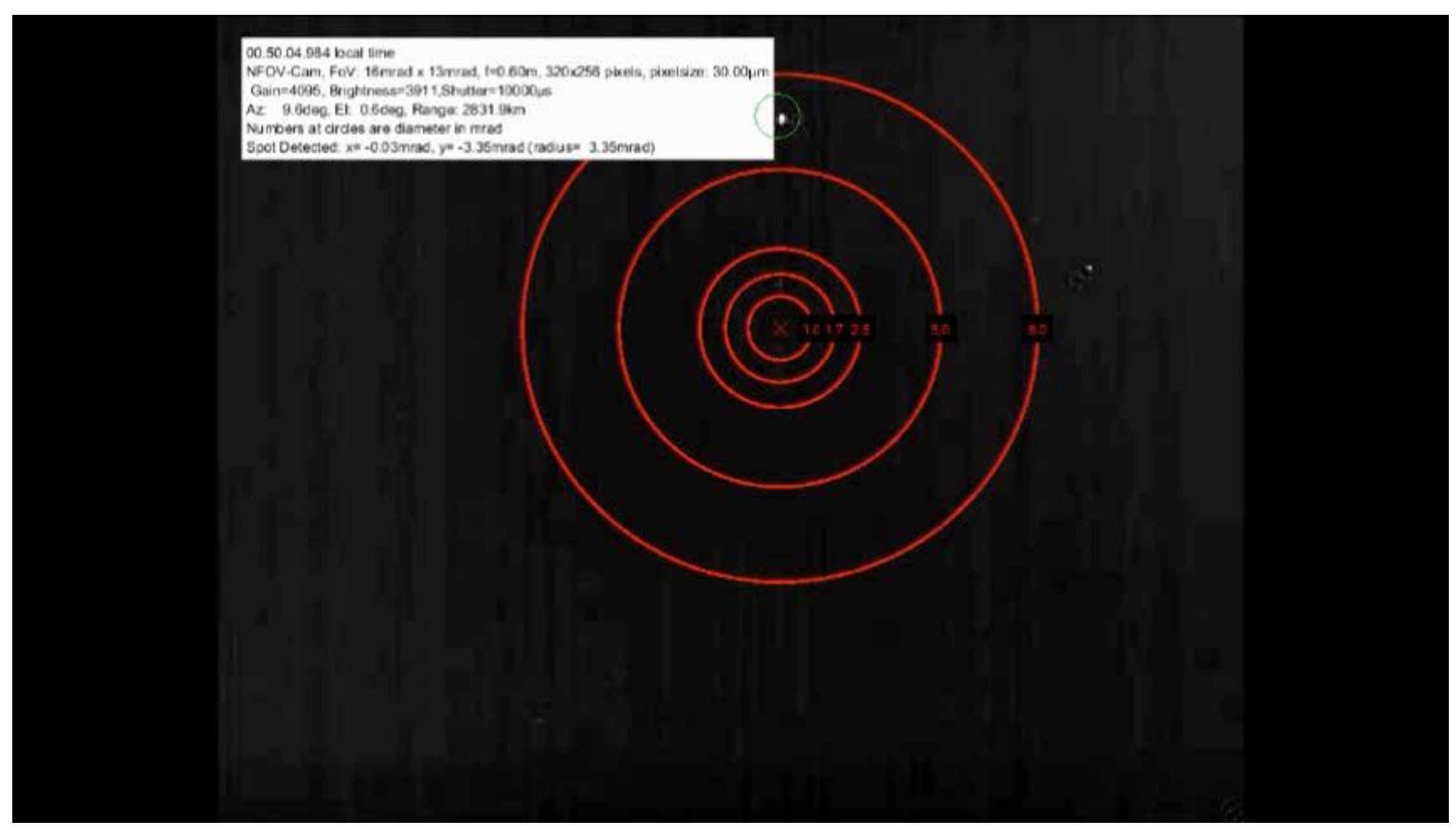


OSIRISv1 on Flying Laptop: First channel measurement (blue: ascending elevation, red: descending elevation)

OSIRISV1 OGS Rx-Signal 21.09.2018

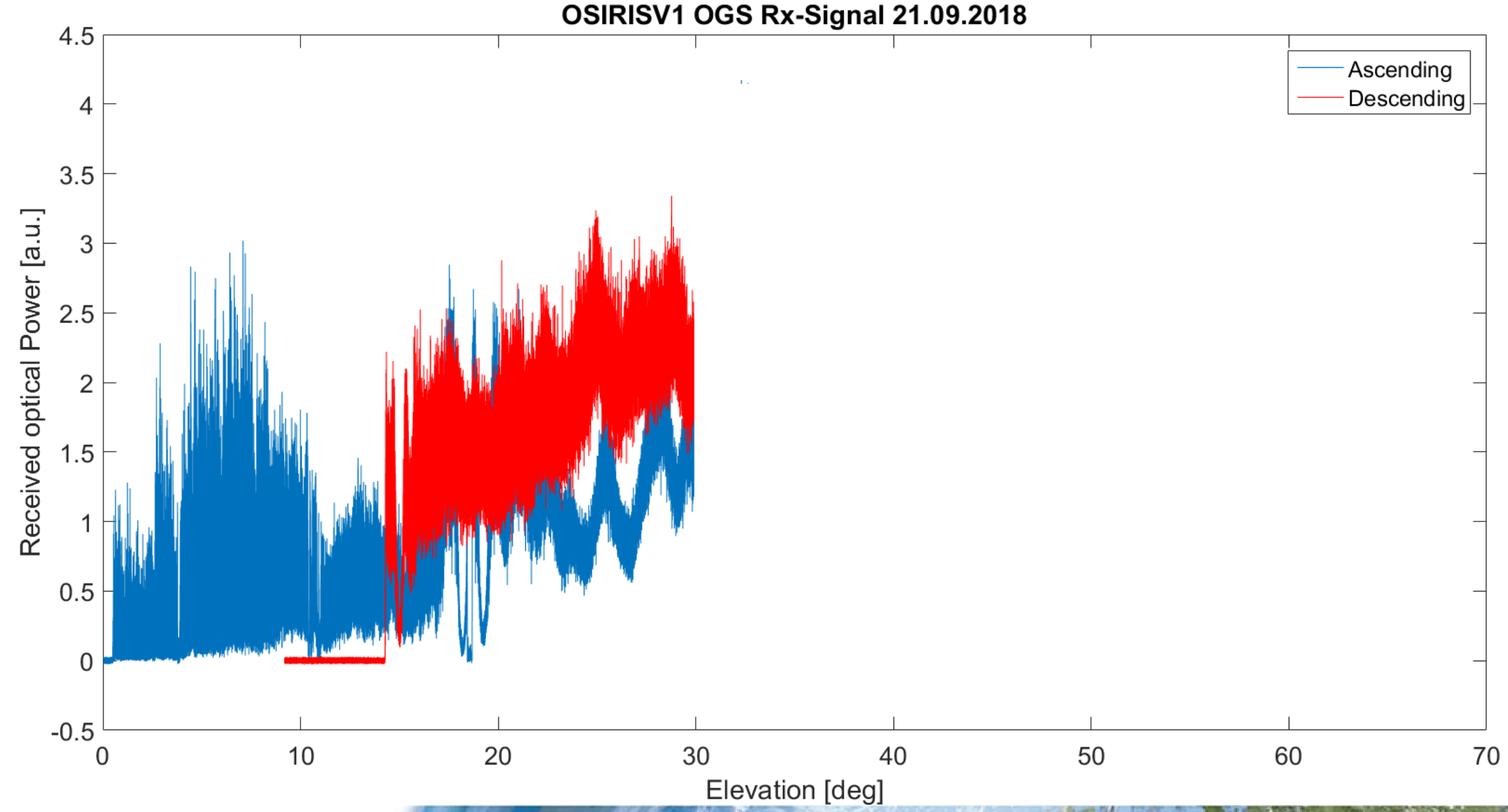




\section{OSIRISv1 on Flying Laptop - Current status and next steps}

- March 2018: First comprehensive campaign with Flying Laptop @ ESA-OGS

- Spring 2018: Further experiments, enhancement of search strategy

- August/September 2018: First signal reception \& angular offset optimization @ DLR-OGS OP

- Achievements so far

- Pure open-loop pointing of satellite (based on Star tracker) with accuracies sufficient for 1.2 mrad beam

- Signal acquisition at 0.4 deg elevation

- 10 minutes continuous signal reception @ 10 minutes OSIRIS operation time

- Currently in preparation / ongoing

- Further channel measurements

- Data reception experiments 
Quantum Key distribution Current Activities: QUBE

- Goal: Cubesat-Terminal for test of quantum communication systems

- With MPL, LMU, Univ. Würzburg, OHB

- Mean optical power: $100 \mathrm{~mW}$

- Downlink data rate: up to $100 \mathrm{Mbit} / \mathrm{s}$

- Beacon wavelength: $1590 \mathrm{~nm}$

- Transmission wavelength: $1550 \mathrm{~nm}$

- Size: 10x10x4 cm3

- Weight: few 100 grams

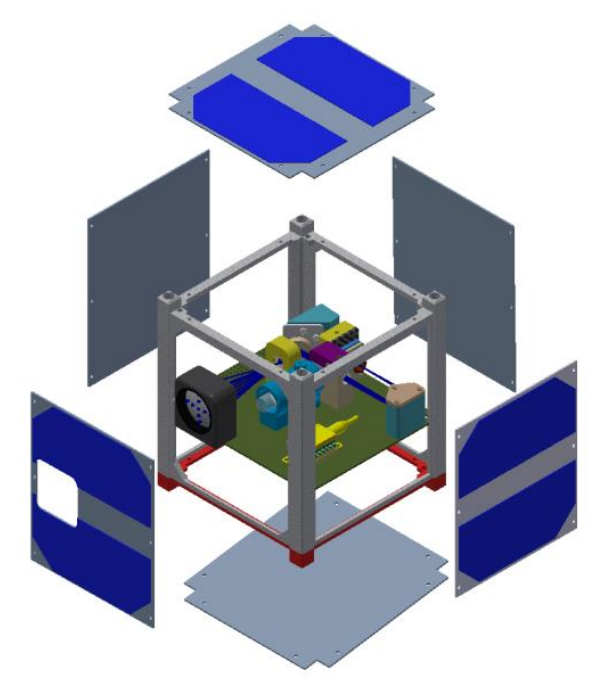

CAD of Cubesat with laser terminal

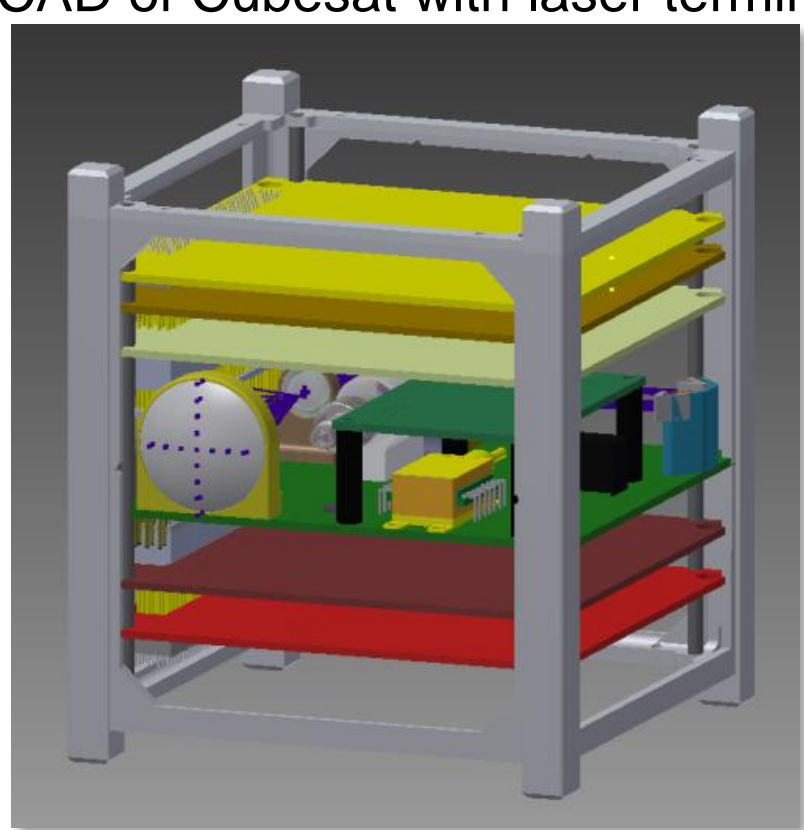

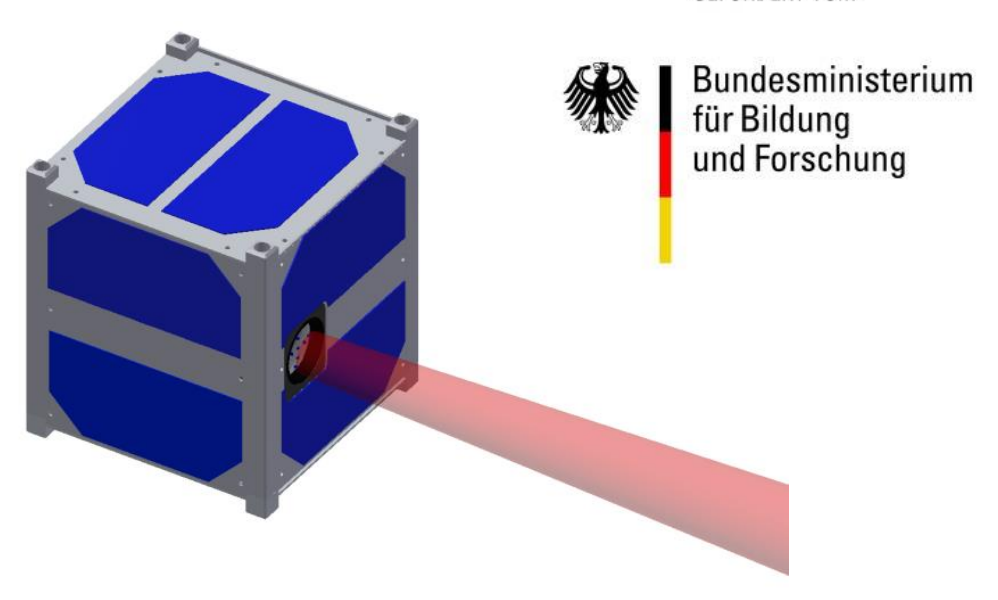

$\mathrm{PCB} /$ laser terminal

LLR LMUJ

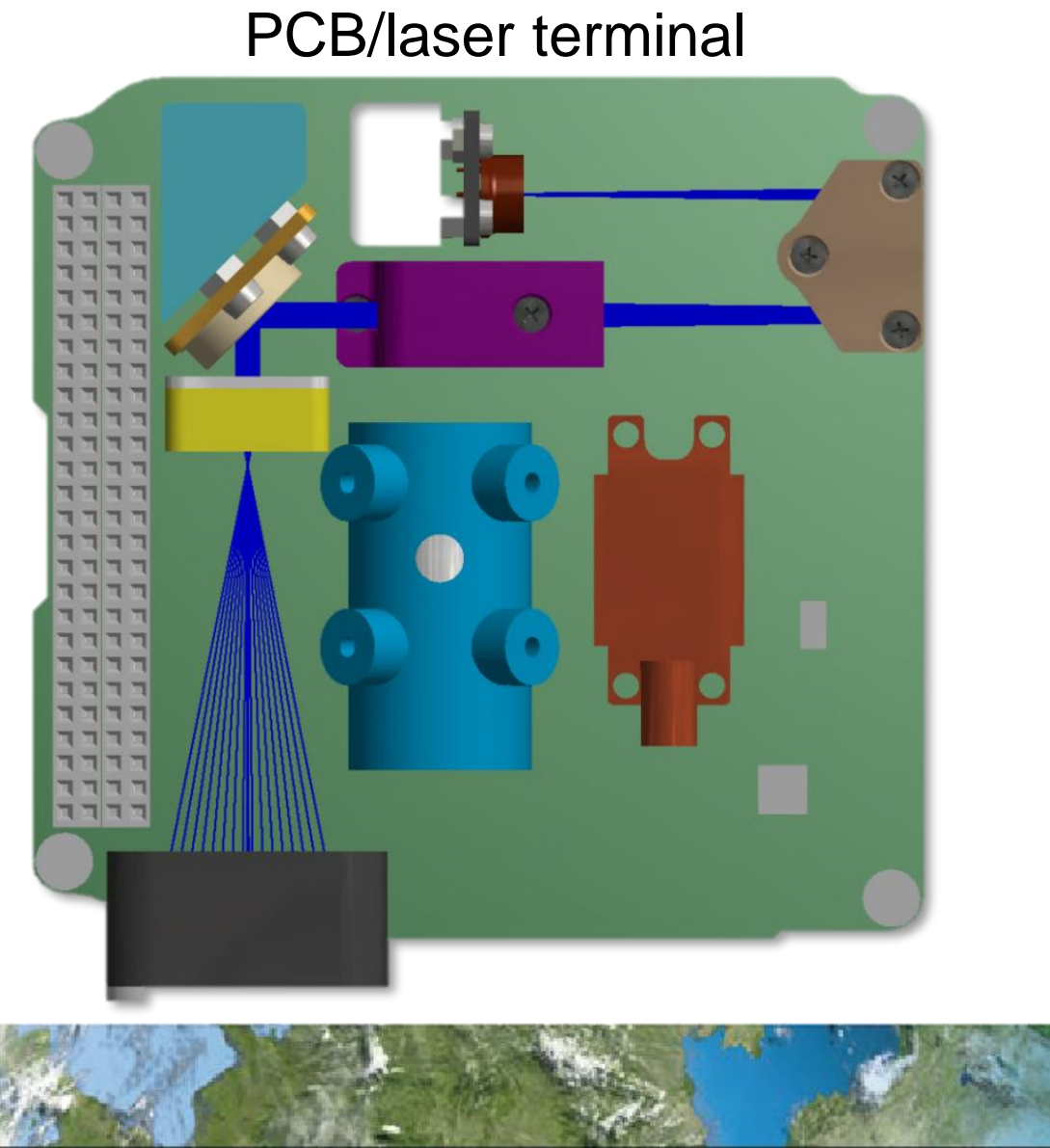




\section{3rd OSIRIS generation (currently in development)}

- Dedicated coarse pointing assembly (CPA) for satellite-independent operation

- Addition of On-Board Storage (>1 Tbyte) and computer system

- Optimized coding with Automatic-Repeat-Requests handled via optical uplink

- System Performance: 10 Gbps, $<5 \mathrm{~kg}$ (Future: Extension to $\underline{N^{*} 10 \mathrm{Gbps} \text { ) }}$

- Reference implementation for upcoming CCSDS standard

- In-Orbit demonstration on Airbus DS Bartolomeo platform / ISS

- Cooperation between Airbus DS / Tesat / DLR

- Experimental validation \& scientific measurements

- Operational phase after IOD
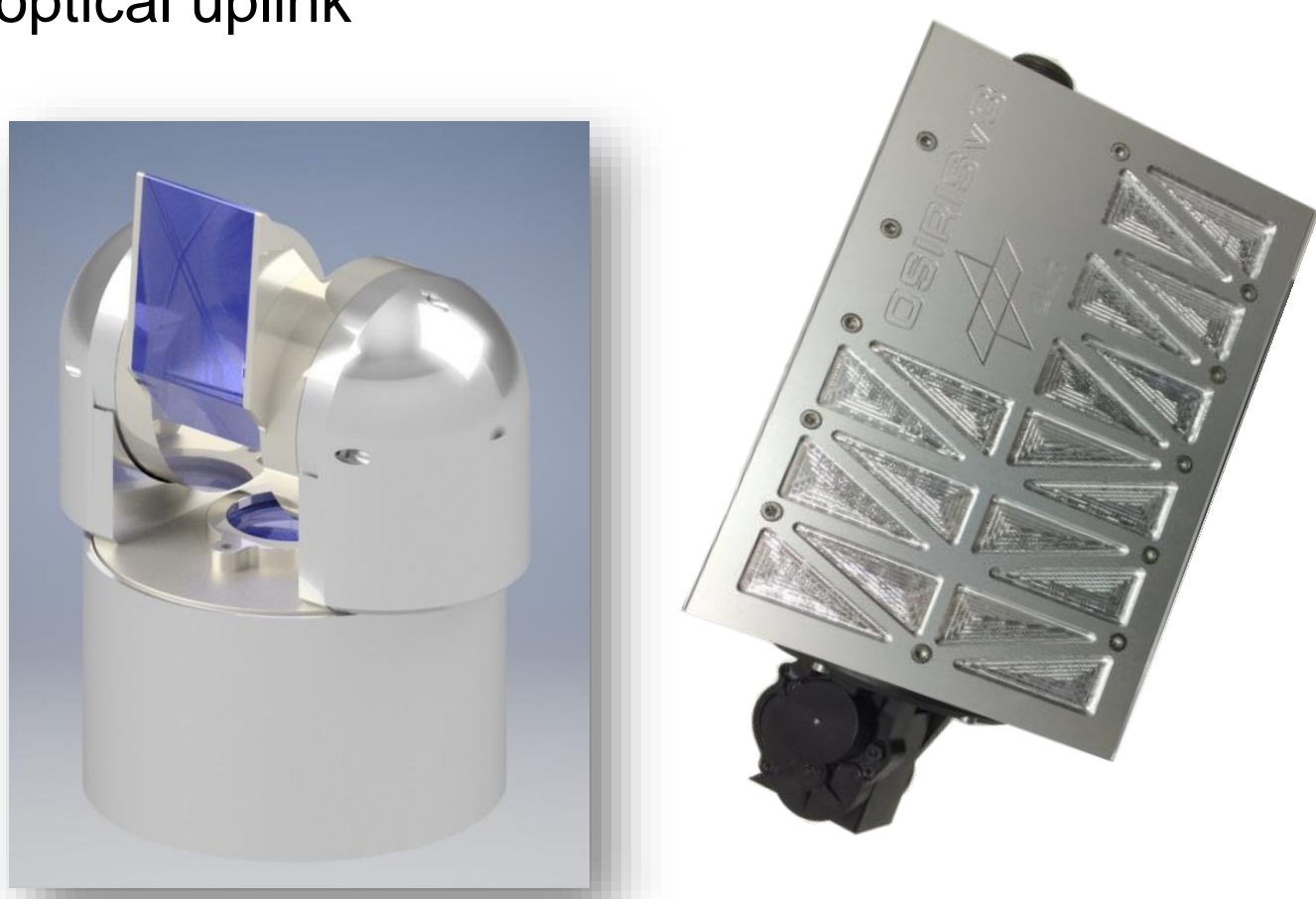
OSIRISv3 on Bartolomeo / ISS

- Cooperation contract signature at ILA 2018 (Airbus DS, Tesat, DLR)
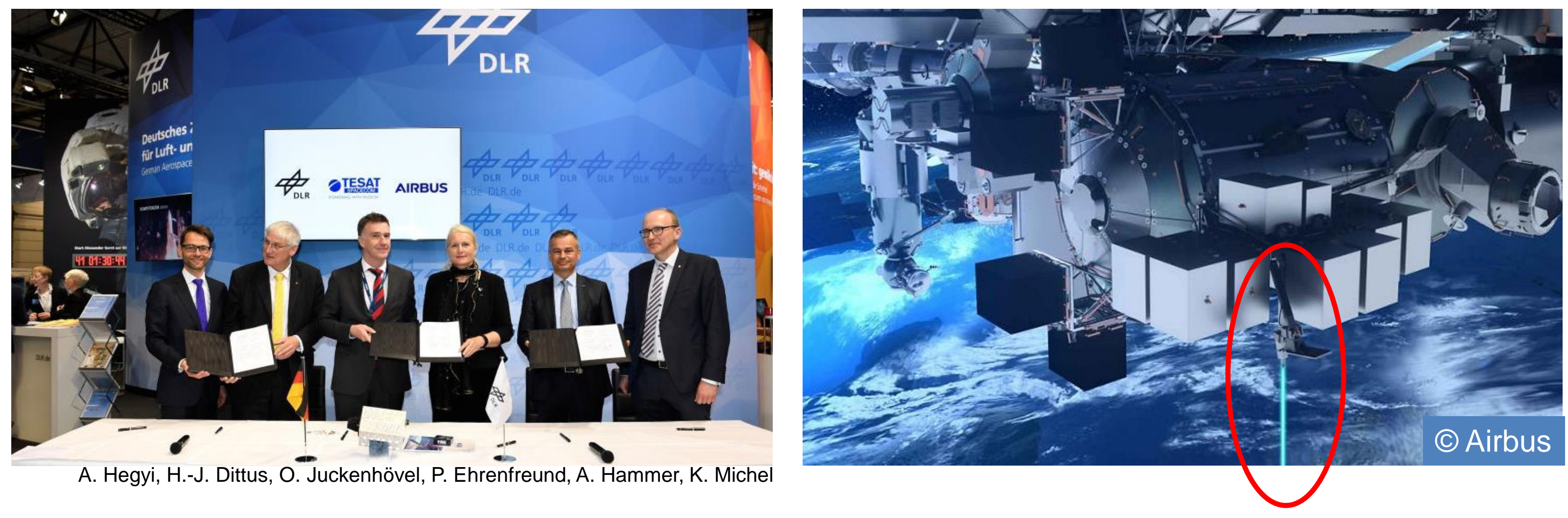

A. Hegyi, H.-J. Dittus, O. Juckenhövel, P. Ehrenfreund, A. Hammer, K. Miche 


\section{QUARTZ-Project}

- Funded by ESA Scylight

- Partners: SES (Prime), MPL, LMU, Tesat, AIT, TNO, IDQ, LUXtrust, itrust consulting, Univ. of Palacky, DLR

- Goal: Develop operational LEO satellite-based QKD system

- Full EM (including processing etc.) by 2020

- Flight demonstration in follow-project by 2022 (tbc)

- Kick-Off on 24th of May, 2018

- Main DLR contributions

- Primeship for satellite payload design

- Extension of OSIRISv3 with QKD-transmitter

- Optical Ground Station for experiments

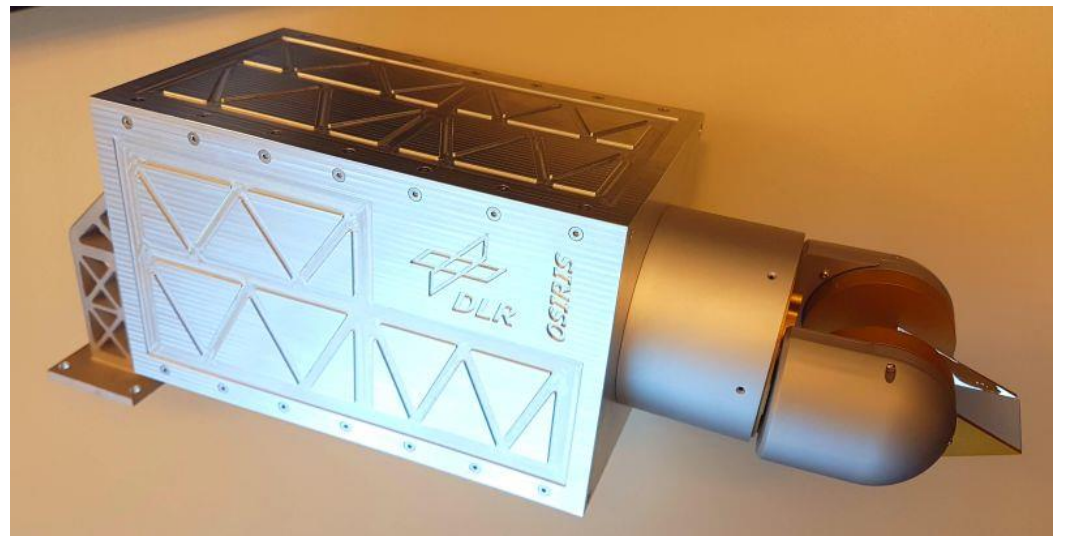




\section{Conclusions}

- OSIRISv1 on Flying Laptop (Univ. of Stuttgart) and OSIRISv2 on BIROS (DLR)

- Both OSIRIS systems are in good health

- Stable signal reception from OSIRISv1 on Flying Laptop

- Further experiments ongoing

- Further developments are ongoing as well

- OSIRIS4Cubesat

- OSIRISv3 for ISS

- Extensions towards Quantum Communications / Quantum Key Distribution ongoing as well 


\section{Status Update on DLR's OSIRIS program}

Christian Fuchs et. al Institute of Communications and Navigation German Aerospace Center (DLR)

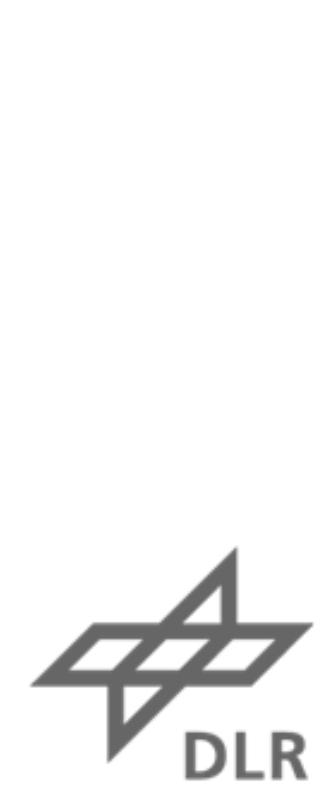




\section{Optical Ground Station Oberpfaffenhofen (OGS-OP)}

- $40 \mathrm{~cm}$ telescope

- 4.5 m clamshell Dome

- Optimized for scientific measurements

- Extension to $80 \mathrm{~cm}$ with Coudé room ongoing
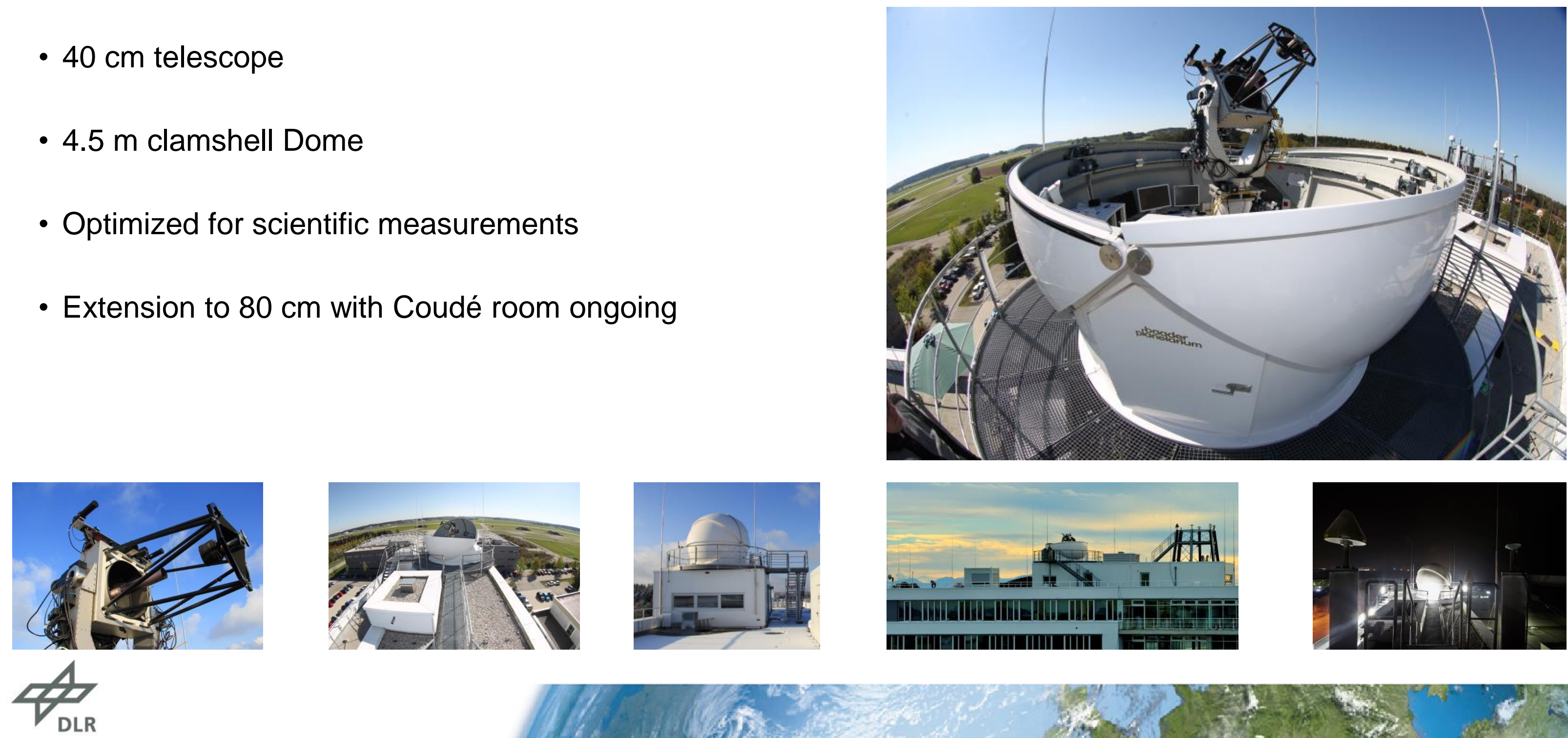


\section{Transportable Optical Ground Station (TOGS)}

- $60 \mathrm{~cm}$ aluminum telescope (manufactured at SHT and polished with partner)

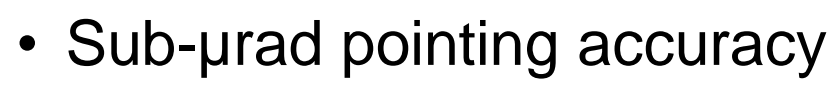
(Torque-motors from Robotics-Spin-Off)

- Optimized for data reception

- Easily transportable to worldwide-locations
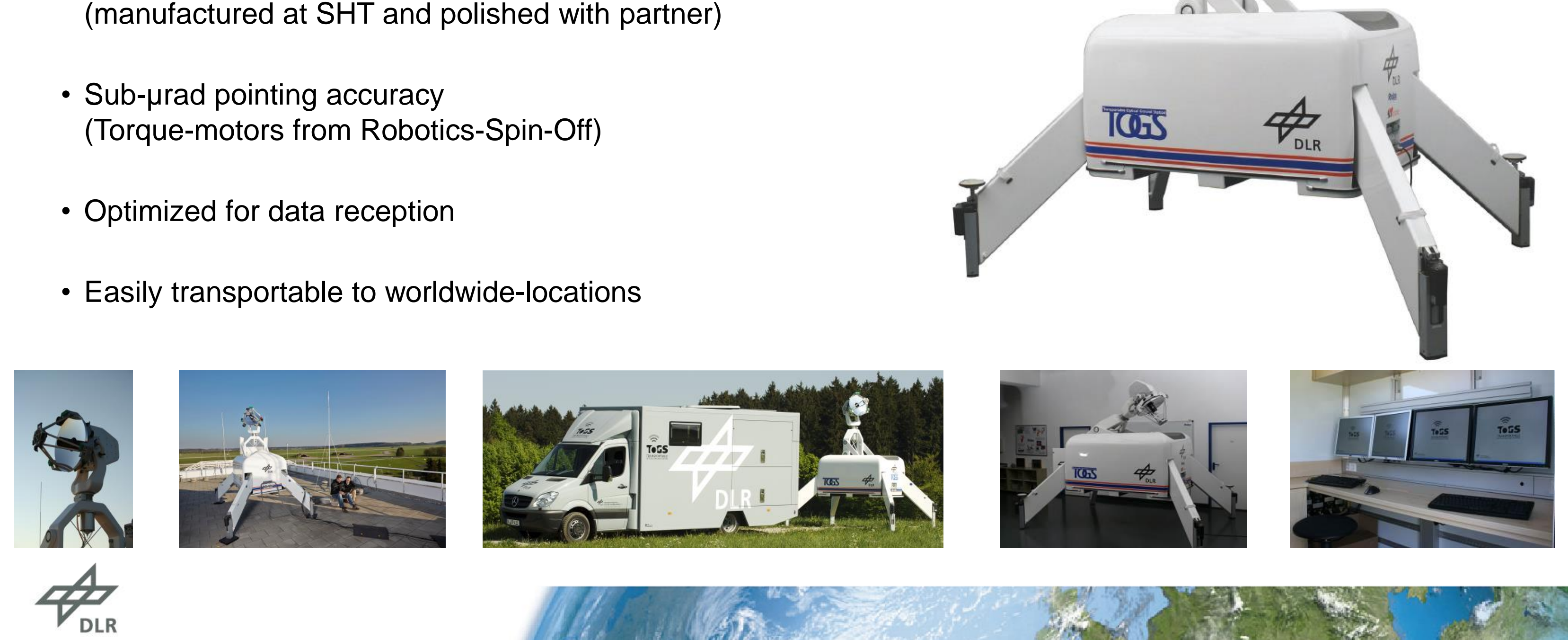